\title{
Approximate controllability of fractional differential equations via resolvent operators
}

\section{Zhenbin Fan*}

\section{*Correspondence:}

jsmathfan@gmail.com

Department of Mathematics, Changshu Institute of Technology,

Suzhou, Jiangsu 215500, China

\begin{abstract}
Of concern are the existence and approximate controllability of fractional differential equations governed by a linear closed operator which generates a resolvent. Using the analytic resolvent method and the continuity of a resolvent in the uniform operator topology, we derive the existence and approximate controllability results of a fractional control system.
\end{abstract}

MSC: 34K37; 47A10; 49J15

Keywords: approximate controllability; analytic resolvent; fractional differential equation

\section{Introduction}

In this paper, we are concerned with the approximate controllability for a fractional differential equation of the form

$$
\left\{\begin{array}{l}
D^{\alpha} x(t)=A x(t)+J_{t}^{1-\alpha}[f(t, x(t))+B u(t)], \quad 0<t \leq b, \\
x(0)=x_{0}
\end{array}\right.
$$

where $D^{\alpha}$ is the Caputo fractional derivative of order $\alpha$ with $0<\alpha<1, A: D(A) \subset X \rightarrow X$ is the infinitesimal generator of a resolvent $S_{\alpha}(t), t \geq 0, B: U \rightarrow X$ is a bounded linear operator, $u \in L^{2}([0, b], U), X$ and $U$ are two real Hilbert spaces, $J_{t}^{1-\alpha} h$ denotes the $1-\alpha$ order fractional integral of $h \in L^{1}([0, b], X)$.

The controllability problem has attracted a lot of mathematicians and engineers' attention since it plays a key role in control theory and engineering and has very important applications in these fields. Many contributions on exact and approximate controllability have been made in recent years. We refer the reader to the recent papers [1-12] and the references therein.

However, there are few articles to study fractional control system (1.1) governed by a linear closed operator which generates a resolvent. The main difficulty is that the resolvent does not have the semigroup property, even the continuity in the uniform operator topology. Fortunately, we can prove the continuity of a resolvent in the uniform operator topology and the compactness of the solution operator in the case of an analytic resolvent. For more details, we refer the reader to the papers $[13,14]$ by Fan and Mophou. A similar idea on the uniform continuity of operators can be found in [15] by Liang, Liu and Xiao. In the present paper, we study approximate controllability of fractional control system (1.1) by using the analytic resolvent method and the uniform continuity of the resolvent.

○2014 Fan; licensee Springer. This is an Open Access article distributed under the terms of the Creative Commons Attribution License (http://creativecommons.org/licenses/by/2.0), which permits unrestricted use, distribution, and reproduction in any medium, provided the original work is properly cited. 
This paper has three sections. In Section 2, we recall some definitions of Caputo fractional derivatives, analytic resolvent, mild solutions to equation (1.1) and the concept of approximate controllability of fractional control systems. In Section 3, we prove the existence and approximate controllability of fractional control system (1.1).

\section{Preliminaries}

Throughout this paper, let $b>0$ be fixed, $\mathbb{N}$ be the set of positive integers. We denote by $(X,\|\cdot\|)$ and $(U,\|\cdot\|)$ two Hilbert spaces, by $C([0, b], X)$ the space of all $X$-valued continuous functions on $[0, b]$ with the norm $\|u\|=\sup \{\|u(t)\|, t \in[0, b]\}$, by $L^{p}([0, b], X)$ the space of $X$-valued Bochner integrable functions on $[0, b]$ with the norm $\|f\|_{L^{p}}=$ $\left(\int_{0}^{b}\|f(t)\|^{p} \mathrm{~d} t\right)^{1 / p}$, where $1 \leq p<\infty$. Also, we denote by $\mathcal{L}(X)$ the space of bounded linear operators from $X$ into $X$ endowed with the norm of operators.

Now, let us recall some basic definitions and results on fractional derivative, resolvent and approximate controllability.

Definition 2.1 ([16]) The fractional order integral of the function $f \in L^{1}([0, b], X)$ of order $\alpha>0$ is defined by

$$
J_{t}^{\alpha} f(t)=\frac{1}{\Gamma(\alpha)} \int_{0}^{t}(t-s)^{\alpha-1} f(s) \mathrm{d} s
$$

where $\Gamma$ is the gamma function.

Definition 2.2 ([16]) The Riemann-Liouville fractional order derivative of order $\alpha$ of a function $f \in L^{1}([0, b], X)$ given on the interval $[0, b]$ is defined by

$$
D_{L}^{\alpha} f(t)=\frac{1}{\Gamma(n-\alpha)} \frac{\mathrm{d}^{n}}{\mathrm{~d} t^{n}} \int_{0}^{t}(t-s)^{n-\alpha-1} f(s) \mathrm{d} s,
$$

where $\alpha \in(n-1, n], n \in \mathbb{N}$.

Definition 2.3 ([16]) The Caputo fractional order derivative of order $\alpha$ of a function $f \in$ $C^{(n)}([0, b], X)$ given on the interval $[0, b]$ is defined by

$$
D^{\alpha} f(t)=\frac{1}{\Gamma(n-\alpha)} \int_{0}^{t}(t-s)^{n-\alpha-1} f^{(n)}(s) \mathrm{d} s,
$$

where $\alpha \in(n-1, n], n \in \mathbb{N}$.

In the remainder of this paper, we always suppose that $0<\alpha<1$ and $A$ is a closed and densely defined linear operator on $X$.

Definition 2.4 ([17]) A family $\left\{S_{\alpha}(t)\right\}_{t \geq 0} \subseteq \mathscr{L}(X)$ of bounded linear operators in $X$ is called a resolvent (or a solution operator) generated by $A$ if the following conditions are satisfied:

(S1) $S_{\alpha}(t)$ is strong continuous on $\mathbb{R}_{+}$and $S_{\alpha}(0)=I$;

(S2) $S_{\alpha}(t) D(A) \subseteq D(A)$ and $A S_{\alpha}(t) x=S_{\alpha}(t) A x$ for all $x \in D(A)$ and $t \geq 0$;

(S3) the resolvent equation holds

$$
S_{\alpha}(t) x=x+\int_{0}^{t} g_{\alpha}(t-s) A S_{\alpha}(s) x \mathrm{~d} s \quad \text { for all } x \in D(A), t \geq 0 .
$$


Since $A$ is a closed and densely defined operator on $X$, it is easy to show that the resolvent equation holds for all $x \in X$ (see [17]).

For $\omega, \theta \in \mathbb{R}$, let

$$
\sum(\omega, \theta):=\{\lambda \in \mathbb{C}:|\arg (\lambda-\omega)|<\theta\} .
$$

Definition 2.5 ([17]) A resolvent $S_{\alpha}(t)$ is called analytic if the function $S_{\alpha}(\cdot): \mathbb{R}_{+} \rightarrow \mathscr{L}(X)$ admits analytic extension to a sector $\sum\left(0, \theta_{0}\right)$ for some $0<\theta_{0} \leq \pi / 2$. An analytic resolvent $S_{\alpha}(t)$ is said to be of analyticity type $\left(\omega_{0}, \theta_{0}\right)$ if for each $\theta<\theta_{0}$ and $\omega>\omega_{0}$, there is $M_{1}=$ $M_{1}(\omega, \theta)$ such that $\|S(z)\| \leq M_{1} e^{\omega \operatorname{Re} z}$ for $z \in \sum(0, \theta)$, where $\operatorname{Re} z$ denotes the real part of $z$.

Definition 2.6 A resolvent $S_{\alpha}(t)$ is called compact for $t>0$ if for every $t>0, S_{\alpha}(t)$ is a compact operator.

Now, we consider the following fractional differential equation

$$
\left\{\begin{array}{l}
D^{\alpha} x(t)=A x(t)+J_{t}^{1-\alpha} f(t), \quad 0<t \leq b, \\
x(0)=x_{0} .
\end{array}\right.
$$

A function $x \in C([0, b], X)$ is called a strong solution of (2.1) if $x(t) \in D(A)$ for all $t \in$ $[0, b], g_{1-\alpha} * x \in C^{1}([0, b], X)$ and $(2.1)$ holds, where $C^{1}([0, b], X)=\left\{x: x^{\prime} \in C([0, b], X)\right\}$, $\left(g_{1-\alpha} * x\right)(t)=\frac{1}{\Gamma(1-\alpha)} \int_{0}^{t}(t-s)^{-\alpha} x(s) \mathrm{d} s$.

A function $x \in C([0, b], X)$ is called an integral solution of (2.1) if $\left(g_{\alpha} * x\right)(t) \in D(A)$ and $x(t)=x_{0}+A\left(g_{\alpha} * x\right)(t)+\int_{0}^{t} f(s) \mathrm{d} s$ for all $t \in[0, b]$.

Suppose that $x_{0} \in X, f \in L^{1}([0, b], X)$ and $x$ is an integral solution of (2.1). Then we can give the following variation of constant formula:

$$
x(t)=S_{\alpha}(t) x_{0}+\int_{0}^{t} S_{\alpha}(t-s) f(s) \mathrm{d} s, \quad 0 \leq t \leq b .
$$

In fact, it follows from the definition of a resolvent and the definition of an integral solution that

$$
\begin{aligned}
1 * x & =\left(S_{\alpha}-A g_{\alpha} * S_{\alpha}\right) * x=S_{\alpha} * x-S_{\alpha} *\left(A g_{\alpha} * x\right) \\
& =S_{\alpha} *\left(x_{0}+1 * f\right)=S_{\alpha} * x_{0}+1 * S_{\alpha} * f,
\end{aligned}
$$

which implies that $x(t)=S_{\alpha}(t) x_{0}+\int_{0}^{t} S_{\alpha}(t-s) f(s) \mathrm{d} s, 0 \leq t \leq b$. That is, the variation of a constant formula is satisfied.

So, we can give the following definition of mild solutions for (1.1).

Definition 2.7 A function $x \in C([0, b], X)$ is called a mild solution of fractional differential equation (1.1) if it satisfies

$$
x(t)=S_{\alpha}(t) x_{0}+\int_{0}^{t} S_{\alpha}(t-s)[f(s, x(s))+B u(s)] \mathrm{d} s, \quad 0 \leq t \leq b,
$$

for $x_{0} \in X$ and $u \in L^{2}([0, b], U)$. 
Let $x$ be a mild solution (state function) of the fractional differential equation corresponding to the control $u$. System (1.1) is said to be approximately controllable on $[0, b]$ if for every desired final state $x_{b} \in X$ and $\varepsilon>0$, there exists a control $u \in L^{2}([0, b], U)$ such that $x$ satisfies $\left\|x(b)-x_{b}\right\|<\varepsilon$. The set

$$
K_{b}(f)=\left\{x(b) \in X: u \in L^{2}([0, b], U), x \text { is the mild solution of (1.1) with control } u\right\}
$$

is called the reachable set of system (1.1).

Definition 2.8 The fractional system is said to be approximately controllable on $[0, b]$ if $\overline{K_{b}(f)}=X$, where $\overline{K_{b}(f)}$ denotes the closure of $K_{b}(f)$.

Now, we introduce the following two relevant operators defined on $X$ :

$$
\begin{aligned}
& \Lambda_{b}=\int_{0}^{b} S_{\alpha}(b-s) B B^{*} S_{\alpha}^{*}(b-s) \mathrm{d} s, \\
& R\left(\lambda, \Lambda_{b}\right)=\left(\lambda I+\Lambda_{b}\right)^{-1}, \quad \lambda>0,
\end{aligned}
$$

where $B^{*}, S_{\alpha}^{*}(b-s)$ denote the adjoint of operators $B$ and $S_{\alpha}(b-s)$, respectively.

In order to find the expression of control $u$ which will be used in the approximate control system, we consider the linear regulator problem consisting of minimizing the cost functional

$$
J(u)=\left\|x(b)-x_{b}\right\|^{2}+\lambda \int_{0}^{b}\|u(t)\|^{2} \mathrm{~d} t,
$$

where $x$ is the solution of (1.1) with control $u, x_{b} \in X, \lambda>0$.

It is known that the control $u$ concerned with approximate controllability of integer order differential equation is just the unique solution of the above optimal problem. Following this idea, we have the following lemma, which can be used to explain the following construction of control function $u$ in (2.4).

Lemma 2.9 Suppose that $u$ is the optimal control of (2.3). Then

$$
u(t)=B^{*} S_{\alpha}^{*}(b-t) R\left(\lambda, \Lambda_{b}\right) p(x(\cdot)), \quad t \in[0, b]
$$

with

$$
p(x(\cdot))=x_{b}-S_{\alpha}(b) x_{0}-\int_{0}^{b} S_{\alpha}(b-s) f(s, x(s)) \mathrm{d} s .
$$

Proof Let $u$ be the optimal control of (2.3). Then $\varepsilon=0$ is a critical point of

$$
I(\varepsilon):=J(u+\varepsilon w)
$$

with $w \in L^{2}([0, b], U)$. By computing the variation of the functional $J$, one has

$$
\left.\left[2\left\langle x(b)-x_{b}, \frac{d}{d \varepsilon}\left(x(b)-x_{b}\right)\right\rangle+2 \lambda \int_{0}^{b}\langle u(t)+\varepsilon w(t), w(t)\rangle_{U} \mathrm{~d} t\right]\right|_{\varepsilon=0}=0,
$$


where $\langle\cdot, \cdot\rangle,\langle\cdot, \cdot\rangle_{U}$ denote the inner products in $X$ and $U$, respectively. Thus,

$$
\begin{aligned}
& \left\langle x(b)-x_{b}, \int_{0}^{b} S_{\alpha}(b-t) B w(t) \mathrm{d} t\right\rangle+\lambda \int_{0}^{b}\langle u(t), w(t)\rangle_{U} \mathrm{~d} t=0, \\
& \int_{0}^{b}\left\langle B^{*} S_{\alpha}^{*}(b-t)\left[x(b)-x_{b}\right], w(t)\right\rangle_{U} \mathrm{~d} t+\lambda \int_{0}^{b}\langle u(t), w(t)\rangle_{U} \mathrm{~d} t=0, \\
& \int_{0}^{b}\left\langle B^{*} S_{\alpha}^{*}(b-t)\left[x(b)-x_{b}\right]+\lambda u(t), w(t)\right\rangle_{U} \mathrm{~d} t=0 .
\end{aligned}
$$

It follows from the arbitrariness of $w$ in $L^{2}([0, b], U)$ that

$$
u(t)=-\lambda^{-1} B^{*} S_{\alpha}^{*}(b-t)\left[x(b)-x_{b}\right]
$$

for almost all $t \in[0, b]$, also for all $t \in[0, b]$ for its continuity in $C([0, b], U)$. Therefore, the state of system (1.1) at a final point $b$ with the above control $u$ is given by

$$
\begin{aligned}
x(b) & =S_{\alpha}(b) x_{0}+\int_{0}^{b} S_{\alpha}(b-s) f(s, x(s)) \mathrm{d} s-\int_{0}^{b} \lambda^{-1} S_{\alpha}(b-s) B B^{*} S_{\alpha}^{*}(b-s)\left[x(b)-x_{b}\right] \mathrm{d} s \\
& =S_{\alpha}(b) x_{0}+\int_{0}^{b} S_{\alpha}(b-s) f(s, x(s)) \mathrm{d} s-\lambda^{-1} \Lambda_{b}\left[x(b)-x_{b}\right] .
\end{aligned}
$$

Let

$$
p(x(\cdot))=x_{b}-S_{\alpha}(b) x_{0}-\int_{0}^{b} S_{\alpha}(b-s) f(s, x(s)) \mathrm{d} s .
$$

Thus,

$$
\begin{aligned}
& x(b)-x_{b}=-p(x(\cdot))-\lambda^{-1} \Lambda_{b}\left[x(b)-x_{b}\right], \\
& x(b)-x_{b}=-\lambda\left(\lambda I+\Lambda_{b}\right)^{-1} p(x(\cdot))=-\lambda R\left(\lambda, \Lambda_{b}\right) p(x(\cdot)) .
\end{aligned}
$$

Consequently,

$$
u(t)=B^{*} S_{\alpha}^{*}(b-t) R\left(\lambda, \Lambda_{b}\right) p(x(\cdot)), \quad t \in[0, b] .
$$

Now, according to Lemma 2.9, for every $\lambda>0$ and $x_{b} \in X$, we construct the following integral system:

$$
\left\{\begin{array}{l}
x(t)=S_{\alpha}(t) x_{0}+\int_{0}^{t} S_{\alpha}(t-s)[f(s, x(s))+B u(s)] \mathrm{d} s, \quad 0 \leq t \leq b, \\
u(t)=B^{*} S_{\alpha}^{*}(b-t) R\left(\lambda, \Lambda_{b}\right) p(x(\cdot)), \\
p(x(\cdot))=x_{b}-S_{\alpha}(b) x_{0}-\int_{0}^{b} S_{\alpha}(b-s) f(s, x(s)) \mathrm{d} s .
\end{array}\right.
$$

In the next section, we will prove the approximate controllability of fractional order system (1.1) by using this integral system. More precisely, we will approximate any fixed point $x_{b} \in X$ under appropriate conditions by using the final state of solution $x$ with the control $u$ given in system (2.4). 


\section{Approximate controllability}

In this section, we first show that for every $\lambda>0$ and $x_{b} \in X$, integral system (2.4) has at least one mild solution. That is, there exists at least one function $x_{\lambda} \in C([0, b], X)$ which satisfies (2.4). Then, we can approximate any point $x_{b}$ in $X$ by using these solutions $\left\{x_{\lambda}\right.$ : $\lambda>0\}$. For this purpose, we need two important lemmas.

Let the Cauchy operator $G: C([0, b], X) \rightarrow C([0, b], X)$ be defined by

$$
(G h)(t)=\int_{0}^{t} S_{\alpha}(t-s) h(s) \mathrm{d} s, \quad t \in[0, b] .
$$

If $S_{\alpha}(t)$ is a compact $C_{0}$-semigroup, it is well known that $G$ is compact. However, it is unknown in the case of a compact resolvent. The main difficulty is that the resolvent does not have the property of semigroups. Thus, it seems to be more complicated to prove the compactness of the Cauchy operator. However, we can prove the continuity of a resolvent in the uniform operator topology in the case of an analytic resolvent, thus the compactness of the Cauchy operator. Moreover, the continuity of a resolvent in the uniform operator topology plays a key role in the proof of the next existence theorem.

Lemma 3.1 ([13, Lemma 10]) Suppose that $S_{\alpha}(t)$ is a compact analytic resolvent of analyticity type $\left(\omega_{0}, \theta_{0}\right)$. Then the following hold:

(i) $\lim _{h \rightarrow 0}\left\|S_{\alpha}(t+h)-S_{\alpha}(t)\right\|=0$ for $t>0$;

(ii) $\lim _{h \rightarrow 0^{+}}\left\|S_{\alpha}(t+h)-S_{\alpha}(h) S_{\alpha}(t)\right\|=0$ for $t>0$;

(iii) $\lim _{h \rightarrow 0^{+}}\left\|S_{\alpha}(t)-S_{\alpha}(h) S_{\alpha}(t-h)\right\|=0$ for $t>0$.

Lemma 3.2 ([13, Lemma 11]) Suppose that $S_{\alpha}(t)$ is a compact analytic resolvent of analyticity type $\left(\omega_{0}, \theta_{0}\right)$. Then the Cauchy operator $G$ defined by (3.1) is a compact operator.

Let $r$ be a fixed positive real number and

$$
W_{r}:=\{x \in C([0, b], X):\|x\| \leq r\}
$$

Clearly, $W_{r}$ is a bounded closed and convex set. We make the following assumptions.

(H1) $S_{\alpha}(t)$ is a compact analytic resolvent of analyticity type $\left(\omega_{0}, \theta_{0}\right)$ and $M=\sup _{t \in[0, b]}\left\|S_{\alpha}(t)\right\|<+\infty$.

(H2) $f:[0, b] \times X \rightarrow X$ is continuous and there exists a positive constant $K$ such that $\|f(t, x)\| \leq K$ for all $(t, x) \in[0, b] \times X$.

(H3) $B: U \rightarrow X$ is a linear bounded operator and there exists $N>0$ such that $\|B\|=N$.

Under these assumptions, we can prove the first main result in this paper. We hereafter always suppose that $\left\|R\left(\lambda, \Lambda_{b}\right)\right\| \leq \frac{1}{\lambda}$ for all $\lambda>0$.

Theorem 3.3 Assume that conditions (H1)-(H3) are satisfied. Then integral system (2.4) has at least one mild solution on $[0, b]$ for every $\lambda>0$ and $x_{b} \in X$.

Proof For fixed $\lambda>0$ and $x_{b} \in X$, we consider the solution operator $Q: C([0, b], X) \rightarrow$ $C([0, b], X)$ defined by

$$
(Q x)(t)=S_{\alpha}(t) x_{0}+\int_{0}^{t} S_{\alpha}(t-s)[f(s, x(s))+B u(s)] \mathrm{d} s, \quad t \in[0, b],
$$


with

$$
\begin{aligned}
& u(t)=B^{*} S_{\alpha}^{*}(b-t) R\left(\lambda, \Lambda_{b}\right) p(x(\cdot)), \\
& p(x(\cdot))=x_{b}-S_{\alpha}(b) x_{0}-\int_{0}^{b} S_{\alpha}(b-s) f(s, x(s)) \mathrm{d} s .
\end{aligned}
$$

It is easy to see that the fixed point of $Q$ is a mild solution of integral system (2.4). Subsequently, we will prove that $Q$ has a fixed point by using Schauder's fixed point theorem.

Firstly, we prove that the mapping $Q$ is continuous on $C([0, b], X)$. For this purpose, let $\left\{x_{n}\right\}_{n \geq 1}$ be a sequence in $C([0, b], X)$ with $\lim _{n \rightarrow \infty} x_{n}=x$ in $C([0, b], X)$. By the continuity of $f$, we obtained that $f\left(s, x_{n}(s)\right)$ converges to $f(s, x(s))$ uniformly for $s \in[0, b]$, and we have

$$
\left\|p\left(x_{n}(\cdot)\right)-p(x(\cdot))\right\| \leq M b \sup _{s \in[0, b]}\left\|f\left(s, x_{n}(s)\right)-f(s, x(s))\right\| .
$$

Thus, for $t \in[0, b]$, we have

$$
\left\|\left(Q x_{n}\right)(t)-(Q x)(t)\right\| \leq\left(M b+\frac{1}{\lambda} M^{3} N^{2} b^{2}\right) \sup _{s \in[0, b]}\left\|f\left(s, x_{n}(s)\right)-f(s, x(s))\right\| \rightarrow 0,
$$

as $n \rightarrow \infty$, which implies that $Q$ is continuous on $C([0, b], X)$.

Secondly, we show that $Q: C([0, b], X) \rightarrow C([0, b], X)$ is a compact operator. According to Lemma 3.2, it is sufficient to prove that $Q_{1}$ is compact, where $Q_{1}: C([0, b], X) \rightarrow$ $C([0, b], X)$ is defined by

$$
\left(Q_{1} x\right)(t)=\int_{0}^{t} S_{\alpha}(t-s) B u(s) \mathrm{d} s, \quad t \in[0, b]
$$

with

$$
\begin{aligned}
& u(t)=B^{*} S_{\alpha}^{*}(b-t) R\left(\lambda, \Lambda_{b}\right) p(x(\cdot)), \\
& p(x(\cdot))=x_{b}-S_{\alpha}(b) x_{0}-\int_{0}^{b} S_{\alpha}(b-s) f(s, x(s)) \mathrm{d} s .
\end{aligned}
$$

Next, we will show that $Q_{1}$ is compact by using the Ascoli-Arzela theorem.

Let $W_{r}$ be any bounded subset of $C([0, b], X)$ (see (3.2)), $0 \leq t_{1} \leq t_{2} \leq b$ and $x \in W_{r}$. We have

$$
\begin{aligned}
& \left\|\left(Q_{1} x\right)\left(t_{2}\right)-\left(Q_{1} x\right)\left(t_{1}\right)\right\| \\
& \quad \leq \int_{0}^{t_{1}}\left\|S_{\alpha}\left(t_{2}-s\right)-S_{\alpha}\left(t_{1}-s\right)\right\|\|B u(s)\| \mathrm{d} s+\int_{t_{1}}^{t_{2}}\left\|S_{\alpha}\left(t_{2}-s\right) B u(s)\right\| \mathrm{d} s \\
& \quad \leq \frac{1}{\lambda} N^{2} M L \int_{0}^{t_{1}}\left\|S_{\alpha}\left(t_{2}-s\right)-S_{\alpha}\left(t_{1}-s\right)\right\| \mathrm{d} s+\frac{1}{\lambda} N^{2} M^{2} L\left(t_{2}-t_{1}\right),
\end{aligned}
$$

where $L=\left\|x_{b}\right\|+M\left\|x_{0}\right\|+M K b$, and $K$ comes from condition (H2).

If $t_{1}=0$, it is easy to see that

$$
\lim _{t_{2} \rightarrow 0}\left\|\left(Q_{1} x\right)\left(t_{2}\right)-\left(Q_{1} x\right)\left(t_{1}\right)\right\|=0 \quad \text { uniformly for } x \in W_{r} .
$$


If $0<t_{1}<b$, for $0<\delta<t_{1}$, we have

$$
\begin{aligned}
&\left\|\left(Q_{1} x\right)\left(t_{2}\right)-\left(Q_{1} x\right)\left(t_{1}\right)\right\| \\
& \leq \frac{1}{\lambda} N^{2} M L\left[\int_{0}^{\delta}\left\|S_{\alpha}\left(t_{2}-s\right)-S_{\alpha}\left(t_{1}-s\right)\right\| \mathrm{d} s+\int_{\delta}^{t_{1}}\left\|S_{\alpha}\left(t_{2}-s\right)-S_{\alpha}\left(t_{1}-s\right)\right\| \mathrm{d} s\right] \\
& \quad+\frac{1}{\lambda} N^{2} M^{2} L\left(t_{2}-t_{1}\right) \\
& \leq \frac{2 \delta}{\lambda} N^{2} M^{2} L+\frac{1}{\lambda} N^{2} M L \int_{\delta}^{t_{1}}\left\|S_{\alpha}\left(t_{2}-s\right)-S_{\alpha}\left(t_{1}-s\right)\right\| \mathrm{d} s+\frac{1}{\lambda} N^{2} M^{2} L\left(t_{2}-t_{1}\right) .
\end{aligned}
$$

Note that from Lemma 3.1 we know that $S_{\alpha}(t)$ is an operator norm continuous uniformly for $[\delta, b]$. Combining this and the arbitrariness of $\delta$ with the above estimation, we can conclude that

$$
\lim _{t_{2} \rightarrow t_{1}}\left\|\left(Q_{1} x\right)\left(t_{2}\right)-\left(Q_{1} x\right)\left(t_{1}\right)\right\|=0 \quad \text { uniformly for } x \in W_{r}
$$

Thus, $Q_{1} W_{r}$ is equicontinuous on $C([0, b], X)$.

Now, for $t=0$, it is easy to see that the set $\left\{\left(Q_{1} x\right)(0): x \in W_{r}\right\}$ is precompact in $X$. Now, let $0<t \leq b$ be given and $0<\varepsilon<t$. Then

$$
\left\{S_{\alpha}(\varepsilon) \int_{0}^{t-\varepsilon} S_{\alpha}(t-s-\varepsilon) B u(s) \mathrm{d} s: x \in W_{r}\right\}
$$

is precompact since $S_{\alpha}(\varepsilon)$ is compact. Moreover, for arbitrary $\varepsilon<\delta<b$, we have

$$
\begin{aligned}
\left\|S_{\alpha}(\varepsilon) \int_{0}^{t-\varepsilon} S_{\alpha}(t-s-\varepsilon) B u(s) \mathrm{d} s-\int_{0}^{t-\varepsilon} S_{\alpha}(t-s) B u(s) \mathrm{d} s\right\| \\
\leq \frac{1}{\lambda} N^{2} M L \int_{0}^{t-\delta}\left\|S_{\alpha}(\varepsilon) S_{\alpha}(t-s-\varepsilon)-S_{\alpha}(t-s)\right\| \mathrm{d} s \\
\quad+\frac{1}{\lambda} N^{2} M L \int_{t-\delta}^{t-\varepsilon}\left\|S_{\alpha}(\varepsilon) S_{\alpha}(t-s-\varepsilon)-S_{\alpha}(t-s)\right\| \mathrm{d} s \\
\leq \frac{1}{\lambda} N^{2} M L \int_{0}^{t-\delta}\left\|S_{\alpha}(\varepsilon) S_{\alpha}(t-s-\varepsilon)-S_{\alpha}(t-s)\right\| \mathrm{d} s+\frac{\delta}{\lambda} N^{2} M L\left(M^{2}+M\right) .
\end{aligned}
$$

From Lemma 3.1(iii), we know

$$
S_{\alpha}(\varepsilon) S_{\alpha}(t-s-\varepsilon)-S_{\alpha}(t-s) \rightarrow 0, \quad \text { as } \varepsilon \rightarrow 0 \text { for } s \in[0, t-\delta]
$$

Then, it follows from the Lebesgue dominated convergence theorem and the arbitrariness of $\delta$ that

$$
\lim _{\varepsilon \rightarrow 0}\left\|S_{\alpha}(\varepsilon) \int_{0}^{t-\varepsilon} S_{\alpha}(t-s-\varepsilon) B u(s) \mathrm{d} s-\int_{0}^{t-\varepsilon} S_{\alpha}(t-s) B u(s) \mathrm{d} s\right\|=0 .
$$


On the other hand,

$$
\begin{aligned}
& \left\|S_{\alpha}(\varepsilon) \int_{0}^{t-\varepsilon} S_{\alpha}(t-s-\varepsilon) B u(s) \mathrm{d} s-\int_{0}^{t} S_{\alpha}(t-s) B u(s) \mathrm{d} s\right\| \\
& \quad \leq\left\|S_{\alpha}(\varepsilon) \int_{0}^{t-\varepsilon} S_{\alpha}(t-s-\varepsilon) B u(s) \mathrm{d} s-\int_{0}^{t-\varepsilon} S_{\alpha}(t-s) B u(s) \mathrm{d} s\right\| \\
& \quad+\left\|\int_{0}^{t-\varepsilon} S_{\alpha}(t-s) B u(s) \mathrm{d} s-\int_{0}^{t} S_{\alpha}(t-s) B u(s) \mathrm{d} s\right\| \\
& \leq\left\|S_{\alpha}(\varepsilon) \int_{0}^{t-\varepsilon} S_{\alpha}(t-s-\varepsilon) B u(s) \mathrm{d} s-\int_{0}^{t-\varepsilon} S_{\alpha}(t-s) B u(s) \mathrm{d} s\right\|+\frac{1}{\lambda} N^{2} M^{2} L \varepsilon .
\end{aligned}
$$

Thus,

$$
\lim _{\varepsilon \rightarrow 0}\left\|S_{\alpha}(\varepsilon) \int_{0}^{t-\varepsilon} S_{\alpha}(t-s-\varepsilon) B u(s) \mathrm{d} s-\int_{0}^{t} S_{\alpha}(t-s) B u(s) \mathrm{d} s\right\|=0,
$$

which implies that $\left\{\left(Q_{1} x\right)(t): x \in W_{r}\right\}$ is precompact in $X$ by using the total boundedness. Thus, $Q_{1}$ is compact in view of the Arzela-Ascoli theorem. Therefore, the solution operator $Q$ is compact.

Finally, we will show that there exists one positive number $r_{0}$ such that $Q W_{r_{0}} \subseteq W_{r_{0}}$. In fact, for all $x \in C([0, b], X)$, it follows from (3.3) that

$$
\|(Q x)\| \leq M\left\|x_{0}\right\|+M K b+\frac{1}{\lambda} N^{2} M^{2} L b .
$$

Then we obtain that for large enough $r_{0}>0$, the inequality $\|(Q x)\| \leq r_{0}$ holds for all $x \in$ $C([0, b], X)$. Thus $Q W_{r_{0}} \subseteq W_{r_{0}}$.

Therefore, by Schauder's fixed point theorem, the operator $Q$ has a fixed point in $W_{r_{0}}$, which is just the mild solution of integral system (2.4).

Next, we present the approximate controllability of fractional control system (1.1). We make the following hypothesis:

(H4) $\lambda R\left(\lambda, \Lambda_{b}\right) \rightarrow 0$ as $\lambda \rightarrow 0^{+}$in the strong operator topology.

Theorem 3.4 Assume that conditions (H1)-(H4) are satisfied. Then fractional control system (1.1) is approximately controllable on $[0, b]$.

Proof According to Theorem 3.3, for every $\lambda>0$ and $x_{b} \in X$, there exists a mild solution $x_{\lambda} \in C([0, b], X)$ such that

$$
x_{\lambda}(t)=S_{\alpha}(t) x_{0}+\int_{0}^{t} S_{\alpha}(t-s)[f(s, x(s))+B u(s)] \mathrm{d} s, \quad t \in[0, b],
$$

with

$$
\begin{aligned}
& u(t)=B^{*} S_{\alpha}^{*}(b-t) R\left(\lambda, \Lambda_{b}\right) p\left(x_{\lambda}(\cdot)\right) \\
& p\left(x_{\lambda}(\cdot)\right)=x_{b}-S_{\alpha}(b) x_{0}-\int_{0}^{b} S_{\alpha}(b-s) f(s, x(s)) \mathrm{d} s .
\end{aligned}
$$


Thus,

$$
\begin{aligned}
x_{\lambda}(b) & =S_{\alpha}(b) x_{0}+\int_{0}^{b} S_{\alpha}(b-s) f\left(s, x_{\lambda}(s)\right) \mathrm{d} s+\Lambda_{b} R\left(\lambda, \Lambda_{b}\right) p\left(x_{\lambda}(\cdot)\right) \\
& =x_{b}-p\left(x_{\lambda}(\cdot)\right)+\Lambda_{b} R\left(\lambda, \Lambda_{b}\right) p\left(x_{\lambda}(\cdot)\right) \\
& =x_{b}-\left(\lambda I+\Lambda_{b}\right) R\left(\lambda, \Lambda_{b}\right) p\left(x_{\lambda}(\cdot)\right)+\Lambda_{b} R\left(\lambda, \Lambda_{b}\right) p\left(x_{\lambda}(\cdot)\right) \\
& =x_{b}-\lambda R\left(\lambda, \Lambda_{b}\right) p\left(x_{\lambda}(\cdot)\right) .
\end{aligned}
$$

Now, by condition (H2), we have

$$
\int_{0}^{b}\left\|f\left(s, x_{\lambda}(s)\right)\right\|^{2} \mathrm{~d} s \leq K^{2} b,
$$

which implies that the sequence $\left\{f\left(\cdot, x_{\lambda}(\cdot)\right): \lambda>0\right\}$ is bounded in the Hilbert space $L^{2}([0, b], X)$. Hence there exists a subsequence of $\left\{f\left(\cdot, x_{\lambda}(\cdot)\right): \lambda>0\right\}$, still denoted by it, converging weakly to some point $\omega(\cdot) \in L^{2}([0, b], X)$. Let

$$
\eta=x_{b}-S_{\alpha}(b) x_{0}-\int_{0}^{b} S_{\alpha}(b-s) \omega(s) \mathrm{d} s .
$$

Thus,

$$
\left\|p\left(x_{\lambda}\right)-\eta\right\| \leq\left\|\int_{0}^{b} S_{\alpha}(b-s)\left[f\left(s, x_{\lambda}(s)\right)-\omega(s)\right] \mathrm{d} s\right\| .
$$

Note that, by using the compactness of $S_{\alpha}(t)$ and Lemma 3.1, similar to the proof of Theorem 3.3, we can prove that the mapping

$$
x(t) \rightarrow \int_{0}^{t} S_{\alpha}(t-s) x(s) \mathrm{d} s
$$

from $L^{2}([0, b], X)$ to $C([0, b], X)$ is compact, i.e., the Cauchy operator $G: L^{2}([0, b], X) \rightarrow$ $C([0, b], X)$ is also compact. So, we obtain that

$$
\int_{0}^{b} S_{\alpha}(b-s)\left[f\left(s, x_{\lambda}(s)\right)-\omega(s)\right] \mathrm{d} s \rightarrow 0 \quad \text { as } \lambda \rightarrow 0^{+}
$$

since $f\left(\cdot, x_{\lambda}(\cdot)\right) \rightarrow \omega(\cdot)$ weakly in $L^{2}([0, b], X)$. Thus, from (3.5) we have

$$
\left\|p\left(x_{\lambda}\right)-\eta\right\| \rightarrow 0 \quad \text { as } \lambda \rightarrow 0^{+} .
$$

In view of (3.4), (3.6) and condition $(\mathrm{H})$, we obtain that

$$
\begin{aligned}
\left\|x_{\lambda}(b)-x_{b}\right\| & \leq\left\|\lambda R\left(\lambda, \Lambda_{b}\right) p\left(x_{\lambda}\right)\right\| \\
& \leq\left\|\lambda R\left(\lambda, \Lambda_{b}\right)\right\| \cdot\left\|p\left(x_{\lambda}\right)-\eta\right\|+\left\|\lambda R\left(\lambda, \Lambda_{b}\right) \eta\right\| \\
& \rightarrow 0 \quad \text { as } \lambda \rightarrow 0^{+},
\end{aligned}
$$

which implies that fractional control system (1.1) is approximately controllable on $[0, b]$. 
Remark 3.5 In the case of a $C_{0}$-semigroup and an integer order derivative, condition (H4) is equivalent to the approximate controllability of the corresponding homogenous linear system. However, due to the complexity of fractional derivatives, one should be more careful to deal with this equivalence. Further discussions on this equivalence and concrete examples will be presented in our consequent papers.

\section{Competing interests}

The author declares that they have no competing interests.

\section{Acknowledgements}

The work was supported by the NSF of China $(11001034,11171210)$ and Jiangsu Overseas Research \& Training Program for University Prominent Young \& Middle-aged Teachers and Presidents.

Received: 30 December 2013 Accepted: 16 January 2014 Published: 03 Feb 2014

\section{References}

1. Ahmed, H: Controllability for Sobolev type fractional integro-differential systems in a Banach spaces. Adv. Differ. Equ. 2012, Article ID 167 (2012)

2. Balachandran, K, Park, JY, Trujillo, J: Controllability of nonlinear fractional dynamical systems. Nonlinear Anal. 75 1919-1926 (2012)

3. Fečkan, M, Wang, J, Zhou, Y: Controllability of fractional functional evolution equations of Sobolev type via characteristic solution operators. J. Optim. Theory Appl. 156(1), 79-95 (2013)

4. Ganesh, R, Sakthivel, R, Mahmudov, NI, Anthoni, SM: Approximate controllability of fractional integrodifferential evolution equations. J. Appl. Math. 2013, Article ID 291816 (2013)

5. Ji, S, Li, G, Wang, M: Controllability of impulsive differential systems with nonlocal conditions. Appl. Math. Comput. 217(16), 6981-6989 (2011)

6. Kumar, S, Sukavanam, N: Approximate controllability of fractional order semilinear systems with bounded delay J. Differ. Equ. 252, 6163-6174 (2012)

7. Mahmudov, Nl: Approximate controllability of fractional Sobolev-type evolution equations in Banach spaces. Abstr. Appl. Anal. 2013, Article ID 502839 (2013)

8. Mahmudov, Nl: Approximate controllability of fractional neutral evolution equations in Banach spaces. Abstr. Appl. Anal. 2013, Article ID 531894 (2013)

9. Mophou, GM, N'Guérékata, GM: Optimal control of a fractional diffusion equation with state constraints. Comput. Math. Appl. 62, 1413-1426 (2011)

10. Rykaczewski, K: Approximate controllability of differential inclusions in Hilbert spaces. Nonlinear Anal. 75, 2701-2712 (2012)

11. Sakthivel, R, Mahmudov, NI, Nieto, JJ: Controllability for a class of fractional-order neutral evolution control systems. Appl. Math. Comput. 218, 10334-10340 (2012)

12. Wang, J, Zhou, Y, Medved, M: On the solvability and optimal controls of fractional integrodifferential evolution systems with infinite delay. J. Optim. Theory Appl. 389, 261-274 (2012)

13. Fan, Z, Mophou, G: Nonlocal problem for fractional differential equations via resolvent operators. Int. J. Differ. Equ 2013, Article ID 490673 (2013)

14. Fan, Z, Mophou, G: Existence and optimal controls for fractional evolution equations. Nonlinear Stud., 20(2), 163-172 (2013)

15. Liang, J, Liu, JH, Xiao, TJ: Nonlocal problems for integrodifferential equations. Dyn. Contin. Discrete Impuls. Syst., Ser. A Math. Anal. 15, 815-824 (2008)

16. Podlubny, I: Fractional Differential Equations. Academic Press, San Diego (1999)

17. Prüss, J: Evolutionary Integral Equations and Applications. Birkhäuser, Basel (1993) 